\title{
Prognostic factors in pediatric pneumococcal meningitis patients in mainland China: a retrospective multicenter study
}

This article was published in the following Dove Press journal: Infection and Drug Resistance

Caiyun Wang, 'Hongmei $\mathrm{Xu}{ }^{2}$ Jikui Deng, ${ }^{3}$ Hui Yu, ${ }^{4}$ Yiping Chen, ${ }^{5}$ Shifu Wang, ${ }^{6}$ Weichun Huang, ${ }^{7}$ Jianhua Hao, ${ }^{8}$ Chun Wang, ${ }^{9}$ Huiling Deng, ${ }^{10}$ Yinghu Chen'

'Infection Disease Department, The Children's Hospital of Zhejiang University School of Medicine, Hangzhou, 310052 , People's Republic of China; ${ }^{2}$ Infection Disease Department, Children's Hospital of Chongqing Medical University, Chongqing 400014, People's Republic of China; ${ }^{3}$ Department of Infectious Diseases, Shenzhen Children's Hospital, Shenzhen 518038, People's Republic of China; ${ }^{4}$ Department of Infectious Diseases, Children's Hospital of Fudan University, Shanghai 201 102, People's Republic of China; ${ }^{5}$ Infection Disease Department, Yuying Children's Hospital of Wenzhou Medica University, Wenzhou 325027, People's Republic of China; 'Department of Children's Medical Laboratory Diagnosis Center, Qilu Children's Hospital of Shandong University, Jinan, 250022, People's Republic of China;

${ }^{7}$ Department of Clinical Laboratory, Shanghai Children's Medical Center of Shanghai Jiaotong University School of Medicine, Shanghai, 200 I27, People's Republic of China; ${ }^{8}$ Infection Disease Department, Kaifeng Children's Hospital, Kaifeng 475000, People's Republic of China; ${ }^{9}$ Clinical Laboratory, Children's Hospital of Shanghai Jiaotong University School of Medicine, Shanghai, 200040, People's Republic of China; ${ }^{10}$ Department of Infectious Diseases, Xi'an Children's Hospital, Xi'an, 710003, People's Republic of China
Background: Prognosis of pneumococcal meningitis (PM) remains very poor, especially in less developed countries. Currently, few multi-centric studies on pediatric PM have been reported in mainland China.

Objectives: This study aimed to explore the correlation of clinical and laboratory findings with complications and prognosis in pediatric PM.

Methods: The pediatric PM patients were retrospectively recruited from ten pediatric tertiary hospitals across China between January 2013 and June 2018. Clinical, biochemical, and microbiological data and follow-up information were collected. Predictive factors for complications and prognostic factors for overall survival (OS) and sequelae-free survival (SFS) were analyzed.

Results: A total of 132 pediatric PM patients were included. Seventy-one patients had complications, 25 patients died, and 39 patients had neurological sequelae. Multivariate logistic regression suggested that age less than 28 months (adjusted OR $=2.654,95 \%$ $\mathrm{CI}=1.067-6.600, P=0.036)$ and lower white blood cells in blood $(\mathrm{aOR}=3.169,95 \%$ $\mathrm{CI}=1.395-7.202, P=0.006)$ were associated with high risk of complications. Multivariate Cox's proportional hazard regression suggested that age less than 28 months (aHR=6.479, 95\%CI $=1.153-36.404, P=0.034)$, coma $(\mathrm{aHR}=9.808,95 \% \mathrm{CI}=2.802-34.323, P=0.000)$, and non-adjuvant steroid therapy $(\mathrm{aHR}=4.76895 \% \mathrm{CI}=1.946-11.678, P=0.001)$ were independent prognostic factors for poor OS; coma $(\mathrm{aHR}=5.841,95 \% \mathrm{CI}=2.652-12.864, P=0.000)$, septic shock on admission $(\mathrm{aHR}=2.949,95 \% \mathrm{CI}=1.049-8.290, P=0.040)$, and lower glucose level in cerebrospinal fluid (CSF) $(\mathrm{aHR}=2.523,95 \% \mathrm{CI}=1.336-4.765, P=0.004)$ were independent prognostic factors for poor SFS.

Conclusion: Age, coma, and adjuvant steroid therapy were independent factors for OS, while coma, septic shock on admission, and lower glucose level in CSF were independent factors for SFS in pediatric PM patients. These factors might be used to identify PM patients with poor prognosis and guide individual treatment.

Keywords: pneumococcal meningitis, clinical findings, complication, prognosis, pediatric

\section{Introduction}

Streptococcus pneumoniae is a common cause of meningitis and pneumococcal meningitis (PM) accounts for $50 \%$ of bacterial meningitis globally. ${ }^{1,2} \mathrm{PM}$ is also a common bacterial meningitis in China. ${ }^{3}$ Most PM patients present with a headache, neck stiffness, and fever. ${ }^{4}$ Culture of cerebrospinal fluid (CSF) obtained from a lumbar puncture should be performed to confirm the diagnosis of PM.
Department of Infection Disease, the Children's Hospital of Zhejiang University School of Medicine, No. 3333, Binsheng Road, Binjiang District, Hangzhou, Zhejiang 310052 , People's Republic of China Tel +861385 715 4891

Email cyh18@zju.edu.cn 
In spite of progress in diagnosis, antibiotic therapy strategies, and intensive care, PM is associated with a mortality rate of $6 \%-17 \%$ in high-income countries including US and some European countries. ${ }^{5-8}$ However, in developing countries and regions such as sub-Saharan Africa, the mortality of PM patients has been reported to be as high as $73 \%{ }^{7,9-13}$ Among surviving patients, $25 \%-$ $63 \%$ suffer from long-term neurological sequelae, ${ }^{5,9,14,15}$ which significantly affect the quality of life of survivors.

Early identification of patients with poor prognosis contributes to individualized treatment and more aggressive therapeutic strategies. A series of clinical features, biochemical, and microbiological factors have been identified as prognostic factors in PM. The most important prognostic factors associated with mortality of PM patients include C-reactive protein, ${ }^{5}$ age, ${ }^{16,17}$ score on SOFA, Glasgow Score, severe hypoglycorrhachia, ${ }^{17}$ corticosteroid treatment, ${ }^{18}$ and glucose in CSF. ${ }^{19}$ And the most important prognostic factors in relation to neurological handicaps seem to be hypoglycorrhachia, ${ }^{20-24}$ shock, coma and convulsions on admission or during hospitalization, ${ }^{25}$ mechanical ventilation requirement, late diagnosis, ataxia, and steroid treatment. ${ }^{26}$

However, these studies are mainly performed in European and American countries and limited to a few research centers. Further multicenter research is required to verify the prognostic value of these factors and improve clinical management of PM patients in mainland China. The present study aimed to recruit pediatric PM patients retrospectively from multiple hospitals in mainland China and investigate the risk factors of complications as well as the prognostic factors of overall survival (OS) and neurological sequelae-free survival (SFS) in pediatric PM patients.

\section{Methods}

\section{Participants}

A multicenter retrospective study was performed to consecutively recruit pediatric PM patients between January 2013 to June 2018 from ten pediatric tertiary hospitals in eight regions in mainland China (Shanghai, Hangzhou, Wenzhou, Xi'an, Chongqing, Shenzhen, Shandong, and Kaifeng).

\section{Patient inclusion and exclusion criteria}

Inclusion criteria: 1) age less than 5 years; 2) compatible with PM diagnosis criteria. Exclusion criteria: 1) patients with known primary immunodeficiency (including humoral immunity disorders, T-cell and B-cell disorders, phagocytic disorders, and complement disorders) or known secondary immunodeficiency (including human immunodeficiency virus infection, nephrotic syndrome, diabetes, etc); 2) patients had no cranial computed tomography (CT) and magnetic resonance imaging (MRI) results. The diagnostic criteria of PM were as follows: 1) clinical signs and symptoms (bulging fontanelles, stiff or painful neck, vomiting, headache, persistent or recurrent fever, change in mental state, seizures, or focal neurologic signs) that comply with meningitis; 2) the results of CSF examination were abnormal (the white blood cell [WBC] count in CSF was more than $10 \times 10^{6}$ / $\mathrm{L}$, in which the neutrophil should be dominant, or the glucose level in CSF was less than $2.78 \mathrm{mmol} / \mathrm{L}$, or the protein level in CSF was more than $0.45 \mathrm{~g} / \mathrm{L} ; 3$ ) positive blood culture for $S$. pneumoniae with positive DNA testing of $S$. pneumoniae in CSF, or positive CSF culture for S. pneumoniae.

\section{Identification of S. pneumoniae isolates}

$S$. pneumoniae isolate was identified by automatic bacterial identification system (VITEK Compact, France) or Optochin Discs (OXOID, UK) according to the National Guide to Clinical Laboratory Procedures.

\section{Data collection}

Case record forms were used to collect data of patients' history, symptoms and signs, laboratory and microbiological findings, clinical course, complications in hospital, vaccination status, treatment, clinical outcome, neurological deficits at discharge, and follow-up visits from the ten hospitals listed in the affiliations. Underlying predisposing factors, including asplenia, history of head trauma or neurosurgery in the past 3 months, anatomical or infectious otorhinolaryngologic disorders, and immunocompromised status, were recorded. Patients with an altered immune status owing to the use of immunosuppressive drugs or splenectomy, diabetes mellitus were considered immunocompromised. Consciousness of the patients was accessed by Glasgow Coma Scale and grouped into normal, decline in consciousness, and coma.

\section{Follow-up}

PM patients were followed-up every month in the first year, and every 6 months thereafter. The last 
follow-up date was September 30, 2018. OS was calculated from the onset date to the time of death of any cause while SFS was calculated from the onset date to the time of neurological deficits or death of any cause. Assessment was performed by a senior, experienced physician. We called the families to come to hospital for a follow-up visit to complete pediatric exam with a potential neuropsychological assessment. A telephonebased interview was suggested to the families who could not come to hospital. Physical examination, Gesell Development Scale test ( $<3$ years and 10 months), Wechsler Intelligence Scale test $(\geq 3$ years and 10 months), and Behavior Questionnaire test ( $\geq 2$ years) were performed. Neurological sequela was evaluated according to the patients' last results of brain MRI, brainstem auditory evoked potential (BAEP), auditory brainstem response, and visual acuity. Neurological deficits included seizures, hemiparesis, ataxia, hydrocephalus and subdural hydrops or empyema, psychomotor retardation, hearing impairment, and visual impairment. Psychomotor retardation was indicated if gross-motor functions (neck control, sitting, walking, running, etc), fine-motor functions (reaching, grasping, drawing, etc), and activities of daily living (toileting, dressing, eating, writing, etc) were affected. Hearing was considered as impaired when the better ear failed to detect a threshold of $40 \mathrm{~dB}$. Visual impairment was noted if the routine ophthalmic examination results of vision, visual field, fundus oculi, and eye movement were abnormal.

\section{Statistics}

Statistical analysis was performed using SPSS 19.0 software (IBM Corporation, Armonk, NY, USA) and GraphPad Prism 6 software (GraphPad Software, Inc., La Jolla, CA, USA). Data were presented as count (\%), or median (interquartile range [IQR]). X-title software was used to obtain the best optimal cut-off values of the continuous variables based on prognosis. Chisquared test and logistic regression analysis were used to investigate the associations of clinical characteristics and laboratory parameters with complications. KaplanMeier curves with logrank test and Cox's proportional hazard regression were applied to assess the associations of clinical characteristics and laboratory parameters with prognosis. $P$-value $<0.05$ was considered significant.

\section{Results}

\section{Baseline clinical characteristics}

Data from one hundred and sixty-one pediatric patients diagnosed with PM were collected from ten pediatric

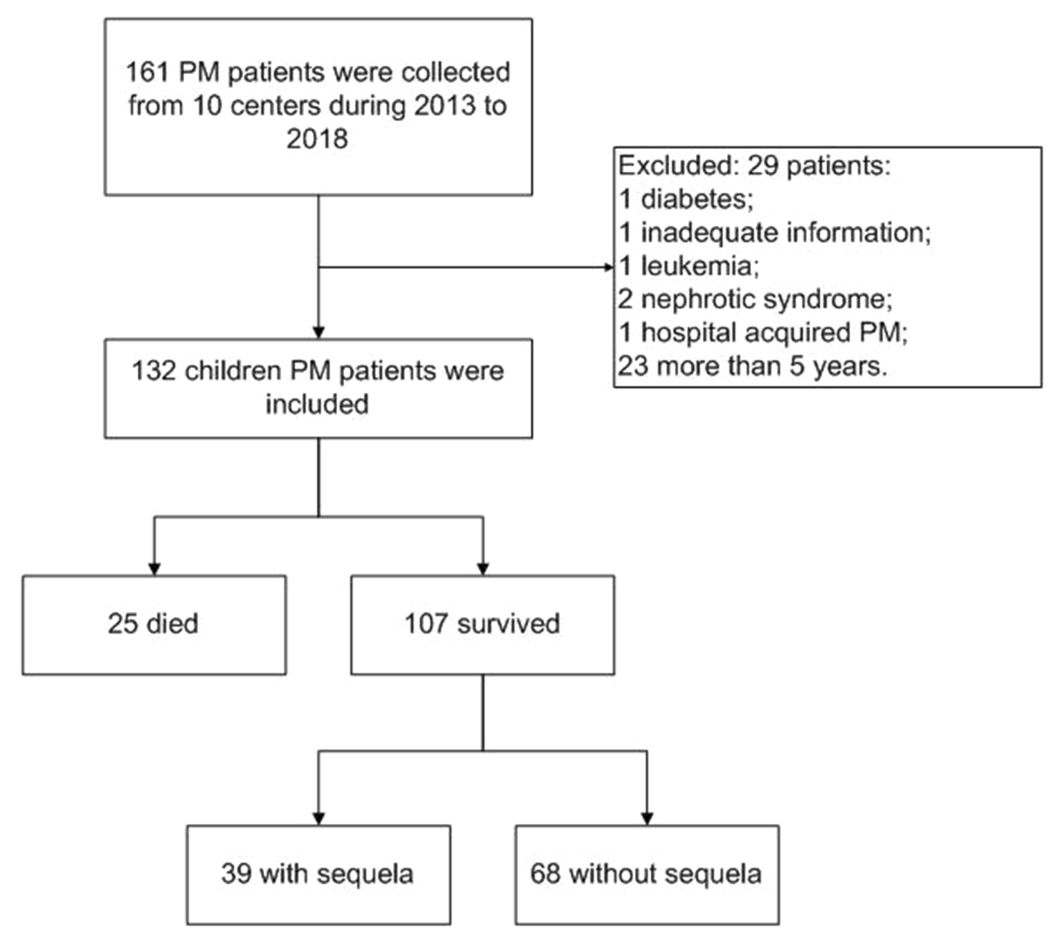

Figure I Flowchart of pediatric pneumococcal meningitis (PM) patients' inclusion. 
Table I Clinical baseline characteristics, laboratory findings, and neurological sequelae of patients with PM

\begin{tabular}{|c|c|c|c|}
\hline Characteristic & Cases n (\%) & Characteristic & Cases n (\%) \\
\hline Total & $132(100)$ & \multirow{6}{*}{$\begin{array}{l}\text { Neurological findings on admission } \\
\text { Triad syndrome } \\
\text { Abnormal mental status } \\
\text { Coma } \\
\text { Convulsions on admission }\end{array}$} & \multirow{6}{*}{$\begin{array}{l}22(16.7) \\
46(34.8) \\
20(15.2) \\
49(37.1)\end{array}$} \\
\hline Sex & & & \\
\hline Male & $88(66.7)$ & & \\
\hline Female & $44(33.3)$ & & \\
\hline Age & \multirow{4}{*}{$\begin{array}{l}13(28) \\
94(7 \mid .2) \\
38(28.8)\end{array}$} & & \\
\hline Median age (IQR, months) & & & \\
\hline$\leq 28$ months & & Laboratory findings & \multirow{4}{*}{$\begin{array}{l}76(57.6) \\
31(23.5) \\
25(I 8.9)\end{array}$} \\
\hline$>28$ months & & Positive CSF culture & \\
\hline Hospital stay & & Positive blood culture & \\
\hline Median time (IQR, days) & $22(18)$ & Positive CSF and blood culture & \\
\hline Symptoms and signs on admission & & WBCs in blood (median, IQR, $\times 10^{9} / \mathrm{L}$ ) & $\mid 4.5(\mid 4.7)$ \\
\hline Headache & $33(25.0)$ & Neutrophil proportion in blood (median, IQR, \%) & $78.5(17.5)$ \\
\hline Nausea & $80(60.6)$ & C-Reactive Protein (median, IQR, mg/L) & $95.8(111.1)$ \\
\hline Neck stiffness & $68(51.5)$ & WBCs in CSF (median, IQR, × $10^{6} / \mathrm{L}$ ) & $400.0(1756.0)$ \\
\hline Bulging fontanelles & $27(20.5)$ & Neutrophil proportion in CSF (median, IQR, \%) & $80.0(12.8)$ \\
\hline Fever & $129(97.7)$ & Protein in CSF (median, IQR, g/L) & $1.4(2.6)$ \\
\hline Septic shock & $7(5.3)$ & Glucose in CSF (median, IQR, mmol/L) & I.I (I.9) \\
\hline Mechanical ventilation & $\mid 7(|| .4)$ & Focal neurological deficits $n=107$ & $39(29.5)$ \\
\hline Multiple organ failure & $5(3.8)$ & Hypophrenia or behavioral deficits & $18(13.6)$ \\
\hline Respiratory failure & $9(6.8)$ & Aphasia & $17(12.9)$ \\
\hline Predisposing factors $^{\mathrm{a}} \mathrm{n}=\mathbf{3 4}$ & $34(25.8)$ & Dyskinesia & $13(9.8)$ \\
\hline Recent or remote cerebral trauma & $\mathrm{II}(8.3)$ & Hearing impairment & $12(9.1)$ \\
\hline CSF leakage & $13(9.8)$ & Hydrocephalus & $8(6.1)$ \\
\hline Previous history of meningitis & $6(4.5)$ & Hemiparesis & $8(6.1)$ \\
\hline Intracranial structure malformation & $5(3.8)$ & Cranial nerve palsies & $5(3.8)$ \\
\hline Previous intracranial surgery ${ }^{\mathrm{b}}$ & $4(3.0)$ & Secondary epilepsy & $4(3.0)$ \\
\hline Craniotomy & $4(3.0)$ & Quadriplegia & $3(2.3)$ \\
\hline Concomitant diseases $n=84$ & $84(63.6)$ & Visual impairment & $2(1.5)$ \\
\hline Pneumonia & $49(37.1)$ & Cerebellar ataxia & $\mathrm{I}(0.8)$ \\
\hline Sinusitis & $22(16.7)$ & Hypothalamus ataxia & $\mathrm{I}(0.8)$ \\
\hline Mastoiditis & $24(18.2)$ & & \\
\hline Otitis & $5(3.8)$ & & \\
\hline Ear or intracranial abnormalities & $8(6.1)$ & & \\
\hline
\end{tabular}

Notes: ${ }^{a}$ Predisposing diseases were defined as previous head trauma, history of intracranial surgery, dura disruption, ear or intracranial abnormalities etc. bintracranial surgery (ventriculostomy, cochlear implantation, ventriculoperitoneal shunt, removal of lateral ventricular reservoir sac, cranioplasty, etc.)

Abbreviations: PM, pneumococcal meningitis; IQR, interquartile range; CSF, cerebrospinal fluid; WBC, white blood cell.

tertiary hospitals across China. Twenty-nine patients were excluded due to primary or secondary immunodeficiency, or age more than 5 years and 132 patients were finally included (Figure 1). As shown in Table 1, of the 132 patients, 88 were male and 44 were female. The median age of the patients was 13 months. Lumbar puncture was performed in all patients. Seventy-sixcases had a positive CSF culture for S. pneumoniae, 31 cases had a positive blood culture for $S$. pneumoniae with a positive DNA testing of
S. pneumoniae in CSF, and 25 cases had both positive CSF culture and positive blood culture for S. pneumoniae. All patients had signs of meningitis.

An amount of $25.8 \%$ (34/132) of patients had predisposing factors for PM including cerebral trauma in eleven (8.3\%), CSF leakage in $13(9.8 \%)$, history of meningitis (ie, the patient had meningitis previously) in six (4.5\%), intracranial structure malformation in five (3.8\%), history of intracranial surgery in four $(3.0 \%)$, and craniotomy in four $(3.0 \%)$ (Table 1). Eighty-four PM patients were 
combined with other concomitant diseases including pneumoniae, mastoiditis, sinusitis, otitis, and ear or intracranial abnormalities. Fever and vomiting were the most frequent symptoms and signs on admission $(97.7 \%$ and $60.6 \%$, respectively). The consciousness status was altered in 46 patients on admission, of which $20(15.2 \%)$ were in a coma.

Laboratory examination results were collected from medical records before or at admission. The medians of WBC counts, protein level in CSF, and glucose level in CSF were $14.5 \times 10^{9} / \mathrm{L} \quad\left(\mathrm{IQR}: 14.7 \times 10^{9} / \mathrm{L}\right), 1.4 \mathrm{~g} / \mathrm{L}$ (IQR: $2.6 \mathrm{~g} / \mathrm{L}$ ), and $1.1 \mathrm{mmol} / \mathrm{L}$ (IQR: $1.9 \mathrm{mmol} / \mathrm{L}$ ), respectively.

\section{Complications and follow-up}

PM complications were found in 53.8\% (71/132) of patients by cranial CT and MRI (Table 2). Subdural effusion, sinusitis or otitis, cerebritis, cerebral edema, hydrocephalus, intracranial hemorrhage, and empyema or abscess were the most common abnormalities and present in $47(35.6 \%), 35(26.5 \%), 29(22.0 \%), 22$ (16.7\%), 17 (12.9\%), 15 (11.4\%), and 12 (9.1\%) patients, respectively. Forty-one patients were associated with more than one abnormality. Twenty-five patients $(18.9 \%)$ died - all during hospitalization. Nine died

Table 2 Complications of 132 pediatric pneumococcal meningitis patients on admission and during hospitalization examined by cranial computed tomography (CT) or magnetic resonance imaging (MRI) for

\begin{tabular}{|l|l|}
\hline Characteristic & Patients $\mathbf{n} \mathbf{( \% )}^{\mathbf{a}}$ \\
\hline Total number of abnormalities $^{\mathrm{b}}$ & $71(53.8)$ \\
Subdural effusion & $47(35.6)$ \\
Sinusitis or otitis & $35(26.5)$ \\
Cerebritis & $29(22.0)$ \\
Cerebral edema & $22(16.7)$ \\
Hydrocephalus & $17(12.9)$ \\
Intracranial hemorrhage & $15(11.4)$ \\
Empyema or abscess & $12(9.1)$ \\
Cerebral atrophy & $7(5.3)$ \\
Skull fracture & $7(5.3)$ \\
Cerebral hernia & $5(3.8)$ \\
Brain infarction & $4(3.0)$ \\
Arachnoid cyst & $3(2.3)$ \\
Other abnormalities &
\end{tabular}

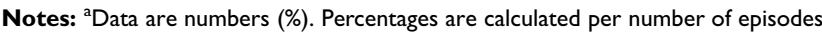
with cranial CT or MRI undertaken. ${ }^{\text {DN }}$ umbers do not add up to totals because of the presence of multiple abnormalities in several patients. 'Middle ear malformation in three, pneumocephalus in two, meningioma in one, and cerebral vascular malformation in one. within 1 week and 16 patients died 1-4 weeks after admission. The predefined neurological sequelae were evaluated in the 107 survivors during follow-up and identified in 39 patients. Hypophrenia or behavioral deficits, aphasia, dyskinesia, hearing impairment, and hydrocephalus were common focal neurological sequelae and present in 18 (13.6\%), 17 (12.9\%), 13 (9.8\%), 12 (9.1\%), and $8(6.1 \%)$ patients of the 107 survivors, respectively (Table 1).

\section{Associations of clinical findings and laboratory parameters with complications}

Chi-squared test was used to investigate the associations of clinical findings and laboratory parameters with complications and the results suggested that age less than 28 months, visual impairment, convulsions on admission, lower WBCs in blood, and predisposing factors were associated with complications in pediatric PM patients (Table 3). Further multivariate logistic regression analysis revealed that age less than 28 months $(\mathrm{aOR}=2.654,95 \%$ $\mathrm{CI}=1.067-6.600, P=0.036)$ and lower WBCs in blood $(\mathrm{aOR}=3.169,95 \% \mathrm{CI}=1.395-7.202, P=0.006)$ were independent predictive factors for complications in pediatric PM patients (Table 4).

\section{Associations of clinical findings and laboratory parameters with accumulating OS}

Kaplan-Meier plot curves and univariate Cox's proportional hazard regression were used to investigate the associations of clinical findings and laboratory parameters with accumulating OS and the results suggested that female sex, age less than 28 months (Figure 2A), coma (Figure 2B), convulsions on admission, respiratory failure, multiple organ dysfunction syndrome (MODS) (omit RF), ventilation, septic shock, non-adjuvant steroid therapy (Figure 2C), and higher protein level in CSF were associated with shorter OS in pediatric PM patients (Table 5). Further multivariate Cox's proportional hazard regression revealed that age less than 28 months $\quad(\mathrm{aHR}=6.479,95 \% \mathrm{CI}=1.153-36.404, \quad P=0.034)$, coma $\quad(\mathrm{aHR}=9.808, \quad 95 \% \mathrm{CI}=2.802-34.323, \quad P=0.000)$, and non-adjuvant steroid therapy $(\mathrm{aHR}=4.768$ 95\% $\mathrm{CI}=1.946-11.678, P=0.001)$ were independent prognostic factors for poor OS in pediatric PM patients (Table 5). 
Table 3 Associations of the clinical features with complications in pediatric PM patients analyzed by chi-squared test

\begin{tabular}{|c|c|c|c|c|c|}
\hline \multirow[t]{2}{*}{ Parameters } & \multirow[t]{2}{*}{ Subgroups } & \multicolumn{2}{|c|}{ Complications } & \multirow[t]{2}{*}{ Chi-squared } & \multirow[t]{2}{*}{$P$-value } \\
\hline & & No & YES & & \\
\hline \multirow[t]{2}{*}{ Sex } & Male & 21 & 23 & 0.061 & 0.805 \\
\hline & Female & 40 & 48 & & \\
\hline \multirow[t]{2}{*}{ Age } & $\leq 28$ months) & 34 & 60 & 13.247 & 0.000 \\
\hline & $>28$ months & 27 & 11 & & \\
\hline \multirow[t]{2}{*}{ Duration of disease } & $\leq 4$ days & 37 & 48 & 0.691 & 0.406 \\
\hline & $>4$ days & 24 & 23 & & \\
\hline \multirow[t]{3}{*}{ Consciousness state } & Normal & 44 & 42 & 2.488 & 0.288 \\
\hline & Decline in consciousness & 10 & 16 & & \\
\hline & Coma & 7 & 13 & & \\
\hline \multirow[t]{2}{*}{ Convulsions on admission } & NO & 46 & 36 & 8.511 & 0.004 \\
\hline & YES & 15 & 35 & & \\
\hline \multirow[t]{2}{*}{ Respiratory failure } & NO & 56 & 67 & 0.339 & 0.560 \\
\hline & YES & 5 & 4 & & \\
\hline \multirow[t]{2}{*}{ MODS } & NO & 59 & 68 & 0.081 & 0.776 \\
\hline & YES & 2 & 3 & & \\
\hline \multirow[t]{2}{*}{ Mechanical ventilation } & NO & 56 & 61 & 1.129 & 0.288 \\
\hline & YES & 5 & 10 & & \\
\hline \multirow[t]{2}{*}{ Septic shock on admission } & NO & 58 & 67 & 0.033 & 0.855 \\
\hline & YES & 3 & 4 & & \\
\hline \multirow[t]{2}{*}{ Adjuvant steroid therapy } & NO & 16 & 18 & 0.013 & 0.909 \\
\hline & YES & 45 & 53 & & \\
\hline \multirow[t]{2}{*}{ Antibiotics treatment } & Early & 15 & 12 & 1.192 & 0.275 \\
\hline & Late & 46 & 59 & & \\
\hline \multirow[t]{2}{*}{ WBCs in blood } & High & 48 & 36 & 11.104 & 0.001 \\
\hline & Low & 13 & 35 & & \\
\hline \multirow[t]{2}{*}{ WBCs in CSF } & High & 15 & 18 & 0.010 & 0.920 \\
\hline & Low & 40 & 50 & & \\
\hline \multirow[t]{2}{*}{ Glucose in CSF } & High & 24 & 28 & 0.000 & 0.991 \\
\hline & Low & 37 & 43 & & \\
\hline \multirow[t]{2}{*}{ Protein in CSF } & Low & 28 & 38 & 1.515 & 0.218 \\
\hline & High & 29 & 25 & & \\
\hline \multirow[t]{2}{*}{ Predisposing factors } & NO & 22 & 12 & 6.301 & 0.012 \\
\hline & YES & 39 & 59 & & \\
\hline \multirow[t]{2}{*}{ Concomitant disease } & NO & 38 & 46 & 0.030 & 0.863 \\
\hline & YES & 22 & 25 & & \\
\hline
\end{tabular}

Note: Bold, indicates $P<0.05$.

Abbreviations: PM, pneumococcal meningitis; MODS, multiple organ dysfunction syndrome; CSF, cerebrospinal fluid; WBC, white blood cell.

Table 4 Associations of the clinical features with complications in pediatric PM patients analyzed by multivariate logistic regression

\begin{tabular}{|l|l|l|l|l|}
\hline Parameters & P-value & OR & \multicolumn{2}{|l|}{$95 \%$ CI } \\
\hline $\begin{array}{l}\text { Age ( } \leq 28 \text { months vs }>28 \\
\text { months) }\end{array}$ & 0.036 & 2.654 & 1.067 & 6.600 \\
Convulsions on admission & 0.156 & 1.823 & 0.795 & 4.181 \\
(YES vs NO) & & & & \\
WBCs in blood (low vs high) & 0.006 & 3.169 & 1.395 & 7.202 \\
Predisposing factors (YES vs & 0.150 & 1.946 & 0.785 & 4.825 \\
NO) & & & & \\
\hline
\end{tabular}

Note: Bold, indicates $P<0.05$.

Abbreviations: PM, pneumococcal meningitis; WBC, white blood cell.

Correlation of clinical findings and laboratory parameters with accumulating SFS

Associations of clinical findings and laboratory parameters with accumulating SFS were also explored. The Kaplan-Meier curves and univariate Cox's proportional hazard regression suggested that coma (Figure 3A), convulsions on admission, respiratory failure, MODS (omit RF), mechanical ventilation, septic shock on admission (Figure 3B), non-adjuvant steroid therapy, 

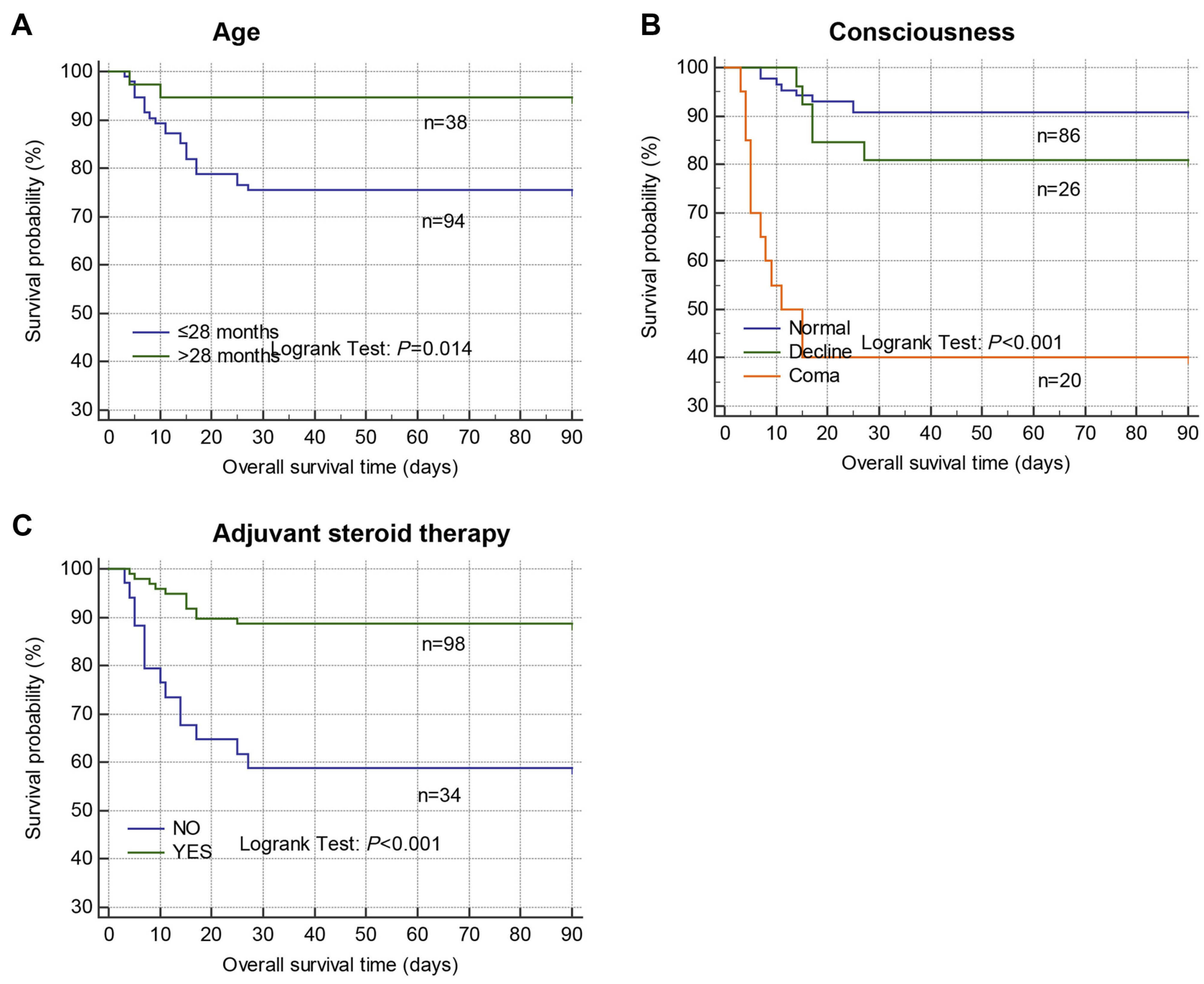

Figure 2 Associations of clinical characteristics with accumulating overall survival in pediatric pneumococcal meningitis (PM) patients were analyzed by Kaplan-Meier plot curves and logrank test. (A) age; (B) consciousness; (C) steroid therapy.

and lower glucose level in CSF (Figure 3C) were associated with shorter SFS (Table 6). After adjusting the confounding factors, coma $\quad(\mathrm{aHR}=5.841, \quad 95 \%$ $\mathrm{CI}=2.652-12.864, P=0.000$ ), septic shock on admission (aHR=2.949, 95\%CI $=1.049-8.290, P=0.040)$, and lower glucose level in CSF $(\mathrm{aHR}=2.523,95 \% \mathrm{CI}=1.336-4.765$, $P=0.004$ ) were found to be independent prognostic factors for unfavorable SFS in multivariate analyses (Table 6).

\section{Discussion}

In the present multicenter study, we included 132 pediatric patients and revealed that the overall mortality of these patients was $18.9 \%$ and the rate of focal neurological sequelae $36.4 \%$, which were higher than that observed in US or European populations. ${ }^{5-8,17,25,27,28}$
A meta-analysis in 1993, including mainly US and European cohorts, revealed a mortality rate of $4.8 \%$ $8.1 \%$ and a neurological handicap rate of $16 \%-26 \%$ in pediatric PM patients. ${ }^{29}$ Another meta-analysis in 2014, performed in Latin American and Caribbean populations, suggested that the mortality rate of pediatric PM patents was $8.3 \% .^{30}$ In developing countries and regions such as sub-Saharan Africa, the mortality of PM patients has been reported to be as high as $73 \% .^{7,9-13}$ The mortality of pediatric PM patients in China was higher than US or European cohorts but lower than African cohorts. The higher mortality and neurological sequelae rates emphasized the need to identify early prognostic factors for pediatric PM patients.

Of the 132 pediatric PM patients in the study, meningitis-associated intracranial complications were found in 
Table 5 Associations of the clinical features with accumulating OS in pediatric PM patients analyzed by Cox's proportional hazard regression

\begin{tabular}{|c|c|c|c|c|c|c|c|c|}
\hline \multirow{3}{*}{$\begin{array}{l}\text { Parameters } \\
\text { Sex (female vs male) }\end{array}$} & \multicolumn{4}{|c|}{ Univariate analysis } & \multicolumn{4}{|c|}{ Multivariate analysis } \\
\hline & \multirow{2}{*}{$\begin{array}{l}P \text {-value } \\
0.010\end{array}$} & \multirow{2}{*}{$\frac{\mathbf{H R}}{2.812}$} & \multicolumn{2}{|c|}{$95 \% \mathrm{Cl}$} & \multirow{2}{*}{$\begin{array}{l}P \text {-value } \\
0.189\end{array}$} & \multirow{2}{*}{$\begin{array}{l}\text { HR } \\
1.774\end{array}$} & \multicolumn{2}{|c|}{$95 \% \mathrm{Cl}$} \\
\hline & & & 1.276 & 6.199 & & & 0.754 & $4.17 \mid$ \\
\hline Age ( $\leq 28$ months vs $>28$ months) & 0.028 & 5.032 & 1.186 & 21.346 & 0.034 & 6.479 & 1.153 & 36.404 \\
\hline Duration of disease ( $>4$ days vs $\leq 4$ days) & 0.189 & 1.692 & 0.772 & 3.709 & & & & \\
\hline Complications (YES vs NO) & 0.473 & 0.750 & 0.342 & 1.645 & & & & \\
\hline Consciousness state & 0.000 & & & & 0.002 & & & \\
\hline Normal & & Ref & & & & Ref. & & \\
\hline Decline in consciousness & 0.202 & 2.071 & 0.678 & 6.332 & 0.079 & 2.916 & 0.882 & 9.643 \\
\hline Coma & 0.000 & 10.641 & 4.321 & 26.201 & 0.000 & 9.808 & 2.802 & 34.323 \\
\hline Convulsions on admission (YES vs NO) & 0.043 & 2.258 & 1.025 & 4.976 & 0.205 & 0.442 & 0.125 & 1.560 \\
\hline Respiratory failure (YES vs NO) & 0.000 & 8.988 & 3.705 & 21.806 & 0.396 & 1.903 & 0.431 & 8.401 \\
\hline MODS (YES vs NO) & 0.001 & 6.363 & 2.170 & 18.659 & 0.326 & 1.808 & 0.555 & 5.888 \\
\hline Mechanical ventilation (YES vs NO) & 0.000 & 5.781 & 2.547 & 13.124 & 0.194 & 2.572 & 0.619 & 10.683 \\
\hline Septic shock on admission (YES vs NO) & 0.000 & 14.465 & 5.519 & 37.914 & 0.053 & 3.927 & 0.983 & 15.693 \\
\hline Adjuvant steroid therapy (NO vs YES) & 0.000 & 4.491 & 2.036 & 9.906 & 0.001 & 4.768 & 1.946 & 11.678 \\
\hline Antibiotics treatment (late vs early) & 0.531 & 1.408 & 0.483 & 4.102 & & & & \\
\hline WBCs in blood (low vs high) & 0.174 & 1.723 & 0.786 & 3.776 & & & & \\
\hline WBCs in CSF (low vs high) & 0.144 & 2.054 & 0.782 & 5.399 & & & & \\
\hline Glucose in CSF (low vs high) & 0.147 & 1.990 & 0.785 & 5.049 & & & & \\
\hline Protein in CSF (high vs low) & 0.034 & 2.536 & 1.074 & 5.984 & 0.953 & 1.039 & 0.292 & 3.693 \\
\hline Concomitant disease (YES vs NO) & 0.065 & 2.512 & 0.943 & 6.696 & & & & \\
\hline Predisposing factors (YES vs NO) & 0.780 & 0.883 & 0.369 & 2.114 & & & & \\
\hline
\end{tabular}

Note: Bold, indicates $P<0.05$.

Abbreviations: OS, overall survivalPM, pneumococcal meningitis; MODS, multiple organ dysfunction syndrome; CSF, cerebrospinal fluid; WBC, white blood cell.

$53.8 \%$ patients by cranial CT or MRI. The most common intracranial complications were subdural effusion, cerebritis, cerebral edema, and hydrocephalus. The prevalence of cerebritis and hydrocephalus in our study was higher than adult PM patients in previous study of. ${ }^{31}$ The prevalence of concomitant diseases was $63.6 \%$ in our study and was similar to the results of previous reports. ${ }^{31-34}$

After multivariate analyses of the prognostic factors in predicting complications, OS, and SFS outcomes in pediatric PM patients, we identified that age less than 28 months was associated with complications $(\mathrm{aOR}=2.654,95 \% \mathrm{CI}=1.067-6.600, P=0.036)$ and short OS $(\mathrm{aHR}=6.479,95 \% \mathrm{CI}=1.153-36.404, P=0.034)$, coma on admission was associated with short OS $(\mathrm{aHR}=9.808$, 95\% $\mathrm{CI}=2.802-34.323, P=0.000)$ and $\mathrm{SFS}(\mathrm{aHR}=5.841$, $95 \% \mathrm{CI}=2.652-12.864, P=0.000$ ), non-adjuvant steroid therapy was associated with short OS $(\mathrm{aHR}=4.768$ $95 \% \mathrm{CI}=1.946-11.678, P=0.001$ ), and septic shock on admission was associated with poor SFS (aHR=2.949, $95 \% \mathrm{CI}=1.049-8.290, P=0.040$ ). Our results were similar to previous reports. ${ }^{16,17,25,26}$ According to previous evidence, the CSF of PM patients usually shows aberrant
WBC count, protein level, and glucose level, thus we also analyzed the prognostic value of WBCs in blood or CSF, protein in CSF, and glucose in CSF to predict complications, OS, and SFS in pediatric PM patients. Our result suggested that lower WBCs in blood was an independent predictive factor of complications and lower glucose level in CSF was an independent prognostic factor of poor SFS. Leukopenia is reflective of severe sepsis, and lower peripheral blood and CSF WBC counts may indicate inadequacy of the immune response. Chao et al also found an association between lower WBCs in CSF and higher mortality in PM. ${ }^{19}$ Lower glucose levels in the CSF is correlated with increased inflammation and cytokine levels ${ }^{35}$ and the inflammatory response is implicated in the pathogenesis of PM and associated brain injury and neuronal death, via releasing potentially cytotoxic agents such as ROS and proteolytic enzymes by leucocytes recruited during the inflammatory response. ${ }^{36}$ A lower glucose level in CSF can indicate increased central nervous system inflammation, which is expected to result in a higher risk of neurological sequelae and poor SFS in PM 

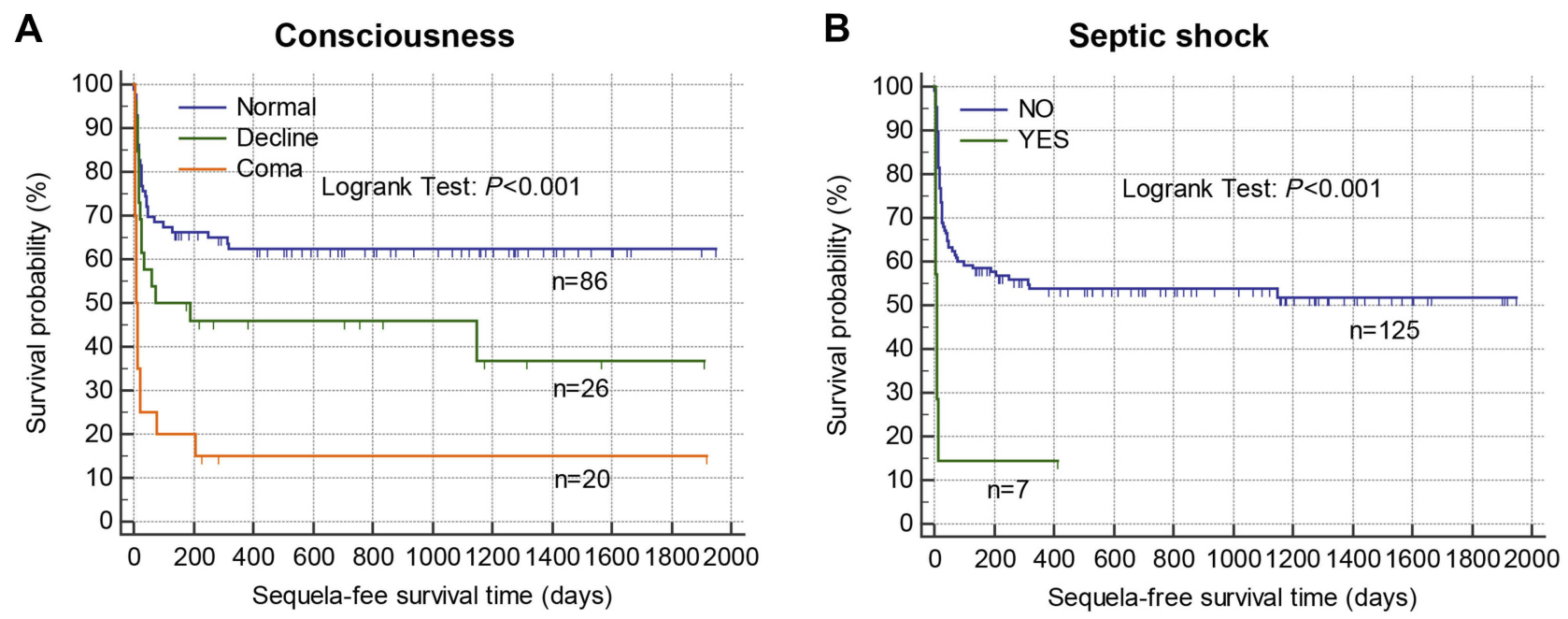

\section{Glucose in CSF}

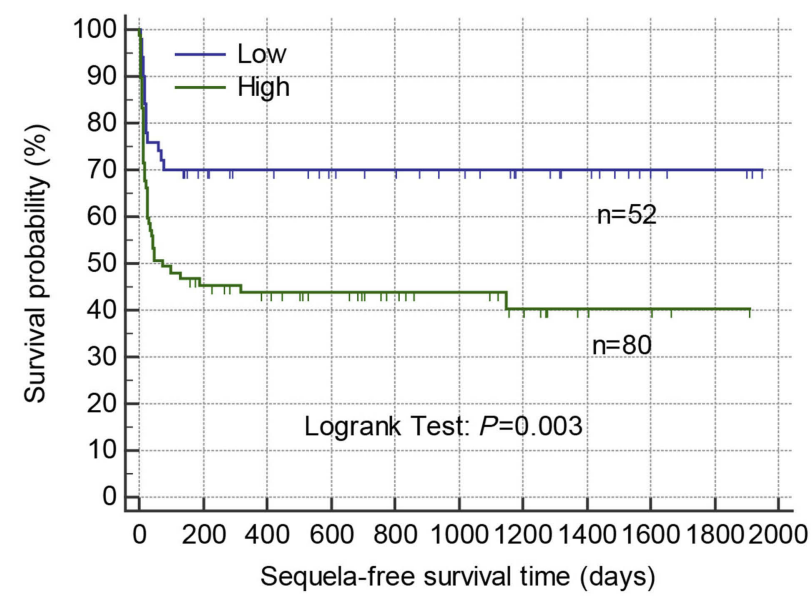

Figure 3 Associations of clinical characteristics and laboratory parameters with accumulating sequelae-free survival in pediatric pneumococcal meningitis (PM) patients were analyzed by Kaplan-Meier plot curves and logrank test. (A) Consciousness; (B) septic shock; (C) glucose in cerebrospinal fluid (CSF).

patients. High protein levels in CSF are related to more severe inflammatory reactions and immune responses during bacterial meningitis, ${ }^{37,38}$ and may also be a predictor of later poor outcomes in PM patients, although we only observed an association between protein level in CSF and OS in univariate analysis.

This study is the most comprehensive nationwide, multi-centric study on pediatric PM in mainland China up to date. However, some limitations still existed in our study. Firstly, information on capsular serotyping of the pediatric PM patients in our retrospective study was not available and the associations of serotype distribution with clinical outcomes of the patients were not clear. Secondly, although pneumococcal vaccines have been licensed in mainland China, none of the patients in our study received pneumococcal vaccination, which might also be associated with prognosis in PM patients.

\section{Conclusion}

Our study showed that PM is a disease with high mortality and neurological sequelae in pediatric patients, and complications during the clinical course occur frequently. In pediatric PM patients, age less than 28 months and lower WBCs in blood were independent predictive factors for complications; age less than 28 months, coma, and non-adjuvant steroid therapy were independent prognostic factors for poor OS; and coma, septic shock on admission, and lower glucose level in CSF were independent prognostic factors for poor SFS. 
Table 6 Associations of the clinical features with accumulating SFS analyzed by Cox's proportional hazard regression

\begin{tabular}{|c|c|c|c|c|c|c|c|c|}
\hline \multirow{3}{*}{$\begin{array}{l}\text { Parameters } \\
\text { Sex (female vs male) }\end{array}$} & \multicolumn{4}{|c|}{ Univariate analysis } & \multicolumn{4}{|c|}{ Multivariate analysis } \\
\hline & \multirow{2}{*}{$\begin{array}{l}P \text {-value } \\
0.521\end{array}$} & \multirow{2}{*}{$\frac{\text { HR }}{1.184}$} & \multicolumn{2}{|c|}{$95 \% \mathrm{Cl}$} & \multirow[t]{2}{*}{$P$-value } & \multirow[t]{2}{*}{ HR } & \multicolumn{2}{|c|}{$95 \% \mathrm{Cl}$} \\
\hline & & & 0.706 & 1.985 & & & & \\
\hline Age ( $\leq 28$ months vs $>28$ months) & 0.181 & 1.485 & 0.832 & 2.650 & & & & \\
\hline Duration of disease ( $>4$ days vs $\leq 4$ days) & 0.064 & 1.593 & 0.973 & 2.609 & & & & \\
\hline Complications (YES vs NO) & 0.457 & 1.208 & 0.735 & 1.984 & & & & \\
\hline Consciousness state & 0.000 & & & & 0.000 & & & \\
\hline Normal & & Ref & & & & Ref & & \\
\hline Decline in consciousness & 0.079 & 1.735 & 0.939 & 3.207 & 0.080 & 1.789 & 0.932 & 3.434 \\
\hline Coma & 0.000 & 4.971 & 2.732 & 9.046 & 0.000 & 5.841 & 2.652 & 12.864 \\
\hline Convulsions on admission (YES vs NO) & 0.050 & 1.638 & 1.001 & 2.683 & 0.915 & 1.034 & 0.559 & 1.912 \\
\hline Respiratory failure (YES vs NO) & 0.000 & 4.337 & 2.037 & 9.233 & 0.566 & 1.413 & 0.433 & 4.607 \\
\hline MODS (YES vs NO) & 0.003 & 4.041 & 1.591 & 10.260 & 0.209 & 1.868 & 0.704 & 4.954 \\
\hline Mechanical ventilation (YES vs NO) & 0.008 & 2.404 & 1.252 & 4.618 & 0.783 & 1.148 & 0.430 & 3.060 \\
\hline Septic shock on admission (YES vs NO) & 0.000 & 5.446 & 2.307 & 12.858 & 0.040 & 2.949 & 1.049 & 8.290 \\
\hline Adjuvant steroid therapy (NO vs YES) & 0.030 & 1.785 & 1.058 & 3.011 & 0.055 & I.77। & 0.989 & 3.173 \\
\hline Antibiotics treatment (late vs early) & 0.425 & 0.794 & 0.450 & 1.399 & & & & \\
\hline WBCs in blood (low vs high) & 0.059 & 1.610 & 0.982 & $2.64 I$ & & & & \\
\hline WBCs in CSF (low vs high) & 0.062 & 1.695 & 0.974 & 2.950 & & & & \\
\hline Glucose in CSF (low vs high) & 0.004 & 2.345 & 1.304 & 4.217 & 0.004 & 2.523 & 1.336 & 4.765 \\
\hline Protein in CSF (high vs low) & 0.318 & 1.301 & 0.777 & 2.178 & & & & \\
\hline Concomitant disease (YES vs NO) & 0.848 & 1.051 & 0.633 & 1.744 & & & & \\
\hline Predisposing factors (YES vs NO) & 0.990 & 0.997 & 0.566 & 1.755 & & & & \\
\hline
\end{tabular}

Note: Bold, indicates $P<0.05$.

Abbreviations: SFS, sequelae-free survival; PM, pneumococcal meningitis; MODS, multiple organ dysfunction syndrome; CSF, cerebrospinal fluid; WBC, white blood cell.

\section{Ethics}

The present study was approved by the Ethics Committee of the Children's Hospital of Zhejiang University School of Medicine. Each PM patient was anonymous in the study and patient/parental consent requirement was waived due to the anonymized data.

\section{Acknowledgments}

We would like to thank Aiwei Lin (Qilu Children's Hospital of Shandong University), Qing Cao (Shanghai Children's Medical Center of Shanghai Jiaotong University School of Medicine), and
Ting Zhang (Children's Hospital of Shanghai Jiaotong University School of Medicine) for re-analyzing the data when revising our manuscript after peer review.

\section{Author contributions}

Caiyun Wang, Hongmei Xu, Jikui Deng, Hui Yu, Yiping Chen, Shifu Wang, Weichun Huang, Jianhua Hao, Chun Wang, Huiling Deng, and Yinghu Chen designed the study. Caiyun Wang and Yinghu Chen drafted the manuscript. All authors contributed to data analysis, drafting and revising the article. All authors 
gave final approval of the version to be published, and agree to be accountable for all aspects of the work.

\section{Disclosure}

The authors report no conflicts of interest in this work.

\section{References}

1. Nuoh RD, Nyarko KM, Nortey P, et al. Review of meningitis surveillance data, upper West Region, Ghana 2009-2013. Pan Afr Med J. 2016;25(Suppl 1):9.

2. Thigpen MC, Whitney CG, Messonnier NE, et al. Bacterial meningitis in the United States, 1998-2007. N Engl J Med. 2011;364 (21):2016-2025. doi:10.1056/NEJMoa1007994

3. Xie Y, Tan Y, Chongsuvivatwong V, et al. A population-based acute meningitis and encephalitis syndromes surveillance in Guangxi, China, May 2007-June 2012. PLoS One. 2015;10(12):e144366. doi:10.1371/journal.pone. 0144366

4. Davis LE. Acute bacterial meningitis. Continuum (Minneap Minn). 2018;24(5, Neuroinfectious Disease):1264-1283.

5. Buchholz G, Koedel U, Pfister HW, Kastenbauer S, Klein M. Dramatic reduction of mortality in pneumococcal meningitis. Crit Care. 2016;20(1):312. doi:10.1186/s13054-016-1362-x

6. Bijlsma MW, Brouwer MC, Kasanmoentalib ES, et al. Communityacquired bacterial meningitis in adults in the Netherlands, 2006-14: a prospective cohort study. Lancet Infect Dis. 2016;16(3):339-347. doi:10.1016/S1473-3099(16)30197-9

7. McGill F, Heyderman RS, Panagiotou S, Tunkel AR, Solomon T. Acute bacterial meningitis in adults. Lancet. 2016;388 (10063):3036-3047. doi:10.1016/S0140-6736(16)30654-7

8. Hasbun R, Rosenthal N, Balada-Llasat JM, et al. Epidemiology of meningitis and encephalitis in the United States, 2011-2014. Clin Infect Dis. 2017;65(3):359-363. doi:10.1093/cid/cix474

9. Stockmann C, Ampofo K, Byington CL, et al. Pneumococcal meningitis in children: epidemiology, serotypes, and outcomes from 1997-2010 in Utah. Pediatrics. 2013;132(3):421-428. doi:10.1542/ peds.2013-0621

10. O'Brien KL, Wolfson LJ, Watt JP, et al. Burden of disease caused by Streptococcus pneumoniae in children younger than 5 years: global estimates. Lancet. 2009;374(9693):893-902. doi:10.1016/S01406736(09)61069-2

11. Gil Prieto R, San Roman Montero J, Gomez Alejandre C, Alvaro Meca LA, Rivero A, Gil de Miguel A. Epidemiology of pneumococcal meningitis hospitalizations in pediatric population in Spain (1998-2006). Vaccine. 2009;27(20):2669-2673.

12. Levy C, Varon E, Picard C, et al. Trends of pneumococcal meningitis in children after introduction of the 13-valent pneumococcal conjugate vaccine in France. Pediatr Infect Dis J. 2014;33(12):1216-1221. doi:10.1097/INF.0000000000000309

13. Mortality GBD, Causes of Death C. Global, regional, and national age-sex specific all-cause and cause-specific mortality for 240 causes of death, 1990-2013: a systematic analysis for the Global Burden of Disease Study 2013. Lancet. 2015;385(9963):117-171. doi:10.1016/ S0140-6736(14)61682-2

14. Lucas MJ, Brouwer MC, van de Beek D. Neurological sequelae of bacterial meningitis. $J$ Infect. 2016;73(1):18-27. doi:10.1016/j. jinf.2016.04.009

15. Dorsett M, Liang SY. Diagnosis and treatment of central nervous system infections in the emergency department. Emerg Med Clin North Am. 2016;34(4):917-942. doi:10.1016/j.emc.2016.06.013

16. Erdem H, Elaldi N, Oztoprak N, et al. Mortality indicators in pneumococcal meningitis: therapeutic implications. Int $J$ Infect Dis. 2014;19:13-19. doi:10.1016/j.ijid.2013.09.012
17. Jordan I, Calzada Y, Monfort L, et al. Clinical, biochemical and microbiological factors associated with the prognosis of pneumococcal meningitis in children. Enferm Infecc Microbiol Clin. 2016;34 (2):101-107. doi:10.1016/j.eimc.2015.03.004

18. Brouwer MC, McIntyre P, Prasad K, van de Beek D. Corticosteroids for acute bacterial meningitis. Cochrane Database Syst Rev. 2015;(9): CD004405.

19. Chao YN, Chiu NC, Huang FY. Clinical features and prognostic factors in childhood pneumococcal meningitis. $J$ Microbiol Immunol Infect. 2008;41(1):48-53.

20. Pagliano P, Fusco U, Attanasio V, et al. Pneumococcal meningitis in childhood: a longitudinal prospective study. FEMS Immunol Med Microbiol. 2007;51(3):488-495. doi:10.1111/j.1574695X.2007.00324.x

21. Irene Koomen DE, Grobbee JJ, Roord RD, Jennekens-Schinkel A, Van Furth AM. Hearing loss at school age in survivors of bacterial meningitis_ assessment,incidence, and prediction. Pediatrics. 2003;112(5):1049-1053. doi:10.1542/peds.112.5.1049

22. McIntyre PB, Macintyre CR, Gilmour R, Wang H. A population based study of the impact of corticosteroid therapy and delayed diagnosis on the outcome of childhood pneumococcal meningitis. Arch Dis Child. 2005;90(4):391-396. doi:10.1136/adc.2004.05 5335

23. Kaplan SL, Goddard J, Van Kleeck M, Catlin FI, Rd. F. Ataxia and deafness in children due to bacterial meningitis. Pediatrics. 1981;68 (1):8-13.

24. Weisfelt M, Hoogman M, van de Beek D, de Gans J, Dreschler WA, Schmand BA. Dexamethasone and long-term outcome in adults with bacterial meningitis. Ann Neurol. 2006;60(4):456-468. doi:10.1002/ ana.20944

25. Kornelisse RF, Westerbeek CML, Spoor AB, et al. Pneumococcal meningitis in children: prognostic indicators and outcome. Clin Infect Dis. 1995;21(6):1390-1397. doi:10.1093/clinids/21.6.1 390

26. Selva L, Ciruela P, Esteva C, et al. Serotype 3 is a common serotype causing invasive pneumococcal disease in children less than 5 years old, as identified by real-time PCR. Eur J Clin Microbiol Infect Dis. 2012;31(7):1487-1495. doi:10.1007/ s10096-011-1468-7

27. Arditi M, Mason EO, Bradley JS, et al. Three-year multicenter surveillance of pneumococcal meningitis in children: clinical characteristics, and outcome related to penicillin susceptibility and dexamethasone use. Pediatrics. 1998;102(5):1087-1097.

28. Buckingham SC, McCullers JA, Luján-Zilbermann J, Knapp KM, Orman KL, English BK. Early vancomycin therapy and adverse outcomes in children with pneumococcal meningitis. Pediatrics. 2006;117(5):1688-1694. doi:10.1542/peds.2005-2282

29. Baraff LJ, Lee SI, Schriger DL. Outcomes of bacterial meningitis in children: a meta-analysis. Pediatr Infect Dis J. 1993;12(5):389-394. doi:10.1097/00006454-199305000-00008

30. Ciapponi A, Elorriaga N, Rojas JI, et al. Epidemiology of pediatric pneumococcal meningitis and bacteremia in Latin America and the caribbean: a systematic review and meta-analysis. Pediatr Infect Dis J. 2014;33(9):971-978. doi:10.1097/ INF.0000000000000309

31. Weisfelt M, van de Beek D, Spanjaard L, Reitsma JB, de Gans J. Clinical features, complications, and outcome in adults with pneumococcal meningitis: a prospective case series. Lancet Neurol. 2006;5(2):123-129. doi:10.1016/S1474-4422(05)70288-X

32. Hoen B, Viel JF, Gérard A, Dureux JB, Canton P. Mortality in pneumococcal meningitis: a multivariate analysis of prognostic factors. Eur J Med. 1993;2(1):28-32.

33. Auburtin M, Porcher R, Bruneel F, et al. Pneumococcal meningitis in the intensive care unit prognostic factors of clinicaloutcome in a series of 80 cases. Am J Respir Crit Care Med. 2002;165 (5):713-717. doi:10.1164/ajrccm.165.8.2106104 
34. Kastenbauer S, Pfister HW. Pneumococcal meningitis in adults: spectrum of complications and prognostic factors in a series of 87 cases. Brain. 2003;126(5):1015-1025. doi:10.1093/brain/awg113

35. Low PS, Lee BW, Yap HK, et al. Inflammatory response in bacterial meningitis: cytokine levels in the cerebrospinal fluid. Ann Trop Paediatr. 1995;15(1):55-59.

36. Mook-Kanamori BB, Geldhoff M, van der Poll T, van de Beek D. Pathogenesis and pathophysiology of pneumococcal meningitis. Clin Microbiol Rev. 2011;24(3):557-591.
37. Kim KS. Acute bacterial meningitis in infants and children. Lancet Infect Dis. 2010;10(1):32-42. doi:10.1016/S1473-3099(09)70306-8

38. Barichello T, Fagundes GD, Generoso JS, Elias SG, Simoes LR, Teixeira AL. Pathophysiology of neonatal acute bacterial meningitis. J Med Microbiol. 2013;62(Pt 12):1781-1789. doi:10. 1099/jmm.0.059840-0

\section{Publish your work in this journal}

Infection and Drug Resistance is an international, peer-reviewed openaccess journal that focuses on the optimal treatment of infection (bacterial, fungal and viral) and the development and institution of preventive strategies to minimize the development and spread of resistance. The journal is specifically concerned with the epidemiology of antibiotic resistance and the mechanisms of resistance development and diffusion in both hospitals and the community. The manuscript management system is completely online and includes a very quick and fair peerreview system, which is all easy to use. Visit http://www.dovepress.com/ testimonials.php to read real quotes from published authors. 